\title{
Photonic Crystal Fiber Temperature Sensor Based on Quantum Dot Nanocoatings
}

\author{
Beatriz Larrión, Miguel Hernáez, Francisco J. Arregui, Javier Goicoechea, \\ Javier Bravo, and Ignacio R. Matías
}

Electrical and Electronic Engineering Department, Public University of Navarre, Campus Arrosadia s/n, 31006 Pamplona, Spain

Correspondence should be addressed to Beatriz Larrión, beatriz.larrion@unavarra.es

Received 3 March 2009; Accepted 16 April 2009

Recommended by Valerio Pruneri

Quantum dot nanocoatings have been deposited by means of the Layer-by-Layer technique on the inner holes of Photonic Crystal Fibers (PCFs) for the fabrication of temperature sensors. The optical properties of these sensors including absorbance, intensity emission, wavelength of the emission band, and the full width at half maximum (FWHM) have been experimentally studied for a temperature range from -40 to $70^{\circ} \mathrm{C}$.

Copyright (C) 2009 Beatriz Larrión et al. This is an open access article distributed under the Creative Commons Attribution License, which permits unrestricted use, distribution, and reproduction in any medium, provided the original work is properly cited.

\section{Introduction}

In the past few years, new optical fibers have been developed in order to get lower nonlinearity, lower attenuation, and unique waveguiding properties. This is the case of Photonic Crystal Fibers (PCFs), also called holey fibers, which contain arrays of tiny air holes along their structure and allow, among other new applications, the fabrication of new optical fiber sensors. In 1995, Birks et al. proposed a fiber with air holes along its length that could guide light through this structure with interesting properties $[1,2]$. Nowadays the PCF has become a subject of extensive research and has opened a new range of possible applications.

The structure of the PCF enables to have different types of fibers such as endless single mode, double clad, germanium or rare earth doped, high birefringence, and many others with particular features due to its manufacturing flexibility. This variety of choices permits the use of PCF in numerous applications such as sensors which measure physical parameters (temperature, pressure, force, etc.), chemical compounds in gas and liquids, and even biosensors [3-7]. In a PCF a fraction of the modal field is located within the holes of the fiber. This allows, by means of the evanescent field interaction, the measurement of different gases, liquids, or biological samples, only if these materials to be measured are placed in the holes of the PCFs. The possibility of coating the holes with sensing materials also exists. However, due to the technical challenge that implies the deposition of thin films on the inner part of the air holes, there is only a small number of works about sensors with this type of sensing coatings $[8,9]$. One of the main contributions presented in this paper is the deposition of a sensing thin film with a thickness of few nanometers inside the holes of a PFC, instead of the classical approach, which consists of filling these holes with the liquid or gas.

More specifically, a PCF temperature sensor based on QD has been fabricated by means of the Layer-by-Layer (LbL) technique for the first time. An experimental study of the optical properties of these QD-based sensors has been performed focused in the optical absorbance, emission intensity, emission peak wavelength, and a feature which had not been studied before in literature, the dependence of the full width at half maximum (FWHM) of QD emission bands with the temperature.

\section{Experimental}

The deposition technique proposed here is the LbL method, technique which enables the building of nanostructured films in surfaces with complex shapes such as, in this case, the walls of the small cylindrical holes of the PCF. The technique is based on the attraction between oppositely charged polyelectrolytes. Its main advantage is the possibility 
of controling the thickness of the coating on the nanometer scale, building the film by creating bilayers of both cationic and anionic solutions consecutively [10-14]. More specifically, in order to fabricate temperature sensing nanofilms inside the holes of the PCF, quantum dots (QDs) have been used as one of the colloids of the deposition process.

QDs are semiconductor nanocrystals with particle diameters typically from 1 to $12 \mathrm{~nm}$, which have also attracted great interest in the last years due to their very appealing optical properties. QDs can be excited in a broad range of wavelengths and, at the same time, have a narrow emission spectrum. Moreover, the center wavelength of the emission peak depends on the geometrical size of the QD; therefore, the emission wavelength can be tuned by changing the size of the nanocrystals. This gives a large choice of emission wavelengths $[12,15,16]$. In fact, QDs have been already proposed for diverse applications such as biological labels, optical sensors, solar cells, photodetectors, lasers, and many other uses [17-24]. In addition, the emission spectrum of QDs changes gradually depending on temperature [25]. The emission intensity decreases, and the emission bands move to higher wavelengths when temperature increases. Due to these features, QDs are an excellent material for temperature sensing [25-28].

2.1. Materials. The polymeric materials used to build the nanostructured coatings were poly(diallyl dimethylammonium chloride) (PDDA) and poly(acrylic acid) (PAA), both purchased from Sigma-Aldrich. Water-based solutions of these materials were prepared at a concentration of $1 \%$ wt., and their pHs were adjusted to 8.0 and 6.0, respectively. These solutions were filtered using $0.45 \mu \mathrm{m}$ pore size syringe filters before starting the film deposition.

The CdSe quantum dots (QDs) used in these experiments have a $5 \mathrm{~nm}$ diameter and emit in the red region of the visible spectrum (620 $\mathrm{nm}$ approximately). These water soluble QDs were supplied by the American Dye Source Inc. and are functionalized with carboxylic groups. The concentration of the QD aqueous solutions was $0.025 \%$ wt, and the $\mathrm{pH}$ was adjusted to 8.0. These solutions were also filtered with a $0.45 \mu \mathrm{m}$ pore diameter filter.

All the solutions were prepared with $18.2 \mathrm{M} \Omega \cdot \mathrm{cm}$ deionized (DI) water from a Barnstead Diamond system.

2.2. Sensor Fabrication. The sensing nanofilms were deposited on the inner air holes of a PCF LMA-20 purchased from Thorlabs Inc. This fiber has a $229 \mu \mathrm{m}$ diameter, and it is optimized for a single mode operation in the $600-1000 \mathrm{~nm}$ wavelength range. The fiber microstructure with the air holes can be observed in the cross section image shown in Figure 1. The diameter of the holes is $6.4 \mu \mathrm{m}$, and the separation between the centers of adjacent holes is $13.2 \mu \mathrm{m}$.

As mentioned in the introduction the LbL technique is used to fabricate the nanofilms on the inner part of the PCF holes. This method is based on the consecutive exposure of the substrate to cationic and anionic solutions in a repetitive sequence. The different charged particles are adsorbed by electrostatic attraction.

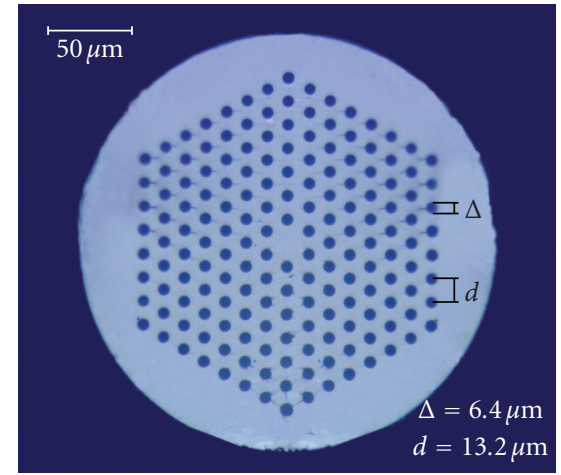

FIgure 1: PCF LMA-20 cross section.

The sensing film has to be coated on the inner part of the PCF, and in order to achieve this, a segment of PCF has to be fixed to a $5 \mathrm{~mL}$ syringe with a $0.5 \mathrm{~mm}$ diameter needle using an epoxy resin. This helps the fluids flow through the segment of fiber and removes the solutions from it. The length of the fiber piece is nearly $6 \mathrm{~cm}$.

To begin the LbL process, a previous bilayer of PDDA and PAA is created on the substrate to enhance the adhesion of the successive layers to the fiber. The segment of the PCF is filled with the cationic PDDA solution with the help of the syringe, and during 3 minutes the solution is left inside the fiber to let the molecules be adsorbed by the inner surface of the microtubes. After the liquid is removed from the fiber, then, the PCF is filled with DI water which removes any rest of the previous solution that could remain still inside the fiber. Once this cleaning process is made, the same procedure is repeated with the PAA solution, which corresponds to the anionic layer.

After the first bilayer, PAA is replaced with QD, and the previously explained process is repeated. The final sensing nanofilm consists of 20 bilayers of PDDA and QD. More details of the LbL process can be found in literature $[10,12-$ $14,26,27,29]$.

When the coating process is finished, the PCF segment is left into a vacuum oven during 15 minutes at $90^{\circ} \mathrm{C}$ in a nitrogen atmosphere. The fiber is then cleaved using a VYTRAN automatic cleaver to obtain smooth cut surfaces in both extremes. A PCF segment of $2.5 \mathrm{~cm}$ with the QD coating is the piece used to make the temperature sensors.

In order to encapsulate the QD film inside the holes of the PCF, the sensor is created by splicing two multimode fibers (MMFs) $(200 \mu \mathrm{m}$ diameter core and $230 \mu \mathrm{m}$ diameter cladding) to the PCF segment. The end of each MMFs is also cut using a Fujikura CT-20 cleaver. An Ericsson FSU905 splicing unit is then used to splice both MMF to the PFC segment. The fusion process with this unit is divided in three parts. The fusion parameters are conveniently selected in order to get a robust fusion between the MMF and the PCF without collapsing the holes of the PCF. Figure 2 shows a microscope picture of the splice between the PCF and the MMF.

Consequently, the QD films deposited on the inner surfaces of the holes are encapsulated as long as they are 


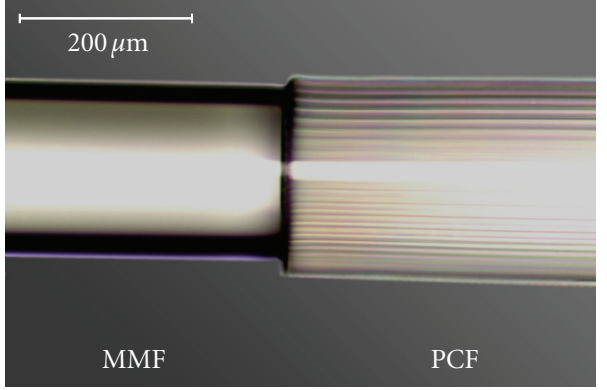

Figure 2: Microscope image of the PCF-MMF splices.

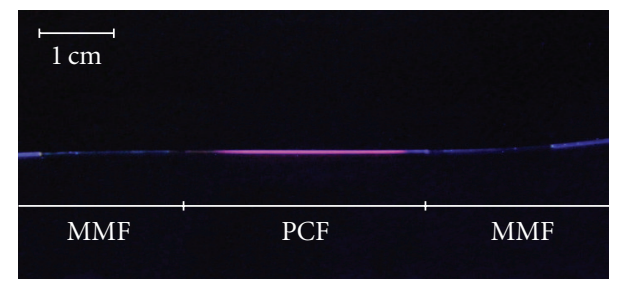

Figure 3: Picture of the LMA20 segment spliced to the MMF fibers under UV illumination. In the middle region the red quantum dots' fluorescence from the PCF inner holes can be seen.

protected against the exterior media and environmental agents such as oxygen. When the sensor fabrication is completed, the fluorescence of QD can be clearly observed by illuminating it with a simple UV-light, as it can be seen in Figure 3.

\section{Results}

In order to study the behavior of the sensors with the temperature, two different sets of experiments have been performed. The first one is intended for the QD absorption spectrum observation and the other one for the study of the emission properties. The setup depicted in Figure 4 was used to perform all the tests. The type of the light source to be used depends on the experiment: a broadband light source is used for the absorption experiments, and a laser is utilized for the emission studies. This light source is connected to a mode scrambler which is used to achieve a stable power by means of avoiding intermodal interference. Then, the PCF sensor is connected in line to this optical fiber. Light from the source passes through the mode scrambler and excites the quantum dots deposited into the sensor. The optical signal from the sensor (the excitation light and the emission light as well) is collected in a spectrometer (USB-2000 FLG, Oceanoptics). Between the sensor and the spectrometer, a high-pass filter is used to limit the excitation signal from the light source which can mask the emission light from the QD.

3.1. Absorption Study. Surprisingly, although optical absorption spectrum is an important QD feature which can also be used for sensing temperature, as was proposed in earliers publications [30], there are few works related to temperature sensors based on QD which study the variation of optical

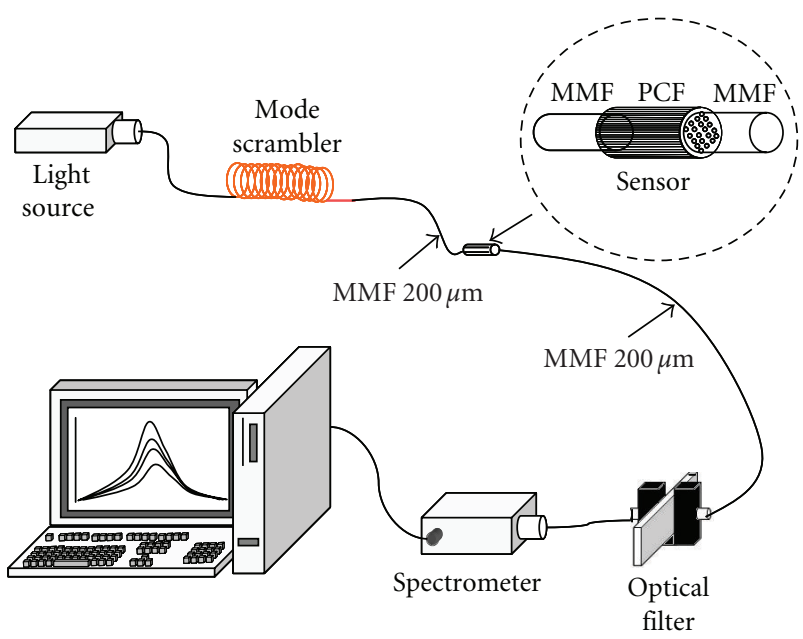

Figure 4: Experimental setup used to collect the optical response from the sensor.

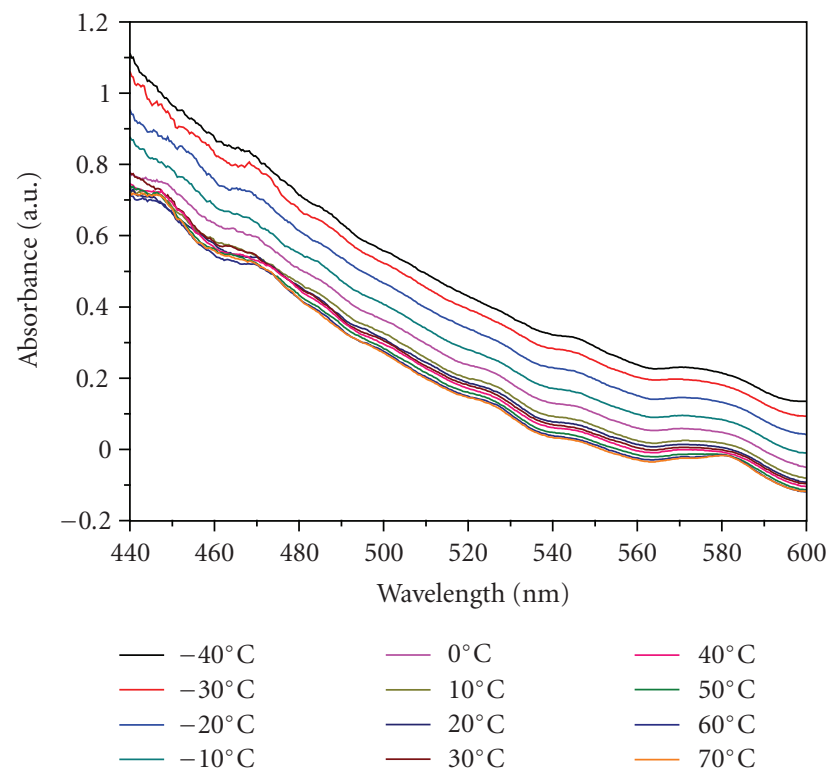

Figure 5: Absorption spectra at different temperatures ( -40 to $\left.+70^{\circ} \mathrm{C}\right)$.

absorption for temperature sensing [31]. In order to measure this parameter, a lowpass optical filter and a deuterium and halogen white light source (Oceanoptics DH 2000) were used in the Figure 4 setup. This source supplies a stable spectrum in the wavelength range from 215 to $1700 \mathrm{~nm}$. The sensor was introduced into a climatic chamber (Angelantoni), and the temperature was varied from -40 to $70^{\circ} \mathrm{C}$. Absorption spectra at different temperatures are shown in Figure 5.

As can be seen in Figure 5, absorbance decreases when temperature rises. In Figure 6, the average absorbance between 500 and $520 \mathrm{~nm}$ at different temperatures is shown.

The experimental data of Figure 6 can be fitted to an exponential curve with a correlation factor of $R^{2}=$ 0.985 . The average sensibility of this measurement method is 0.0027 a.u. $/{ }^{\circ} \mathrm{C}$, and its value in the worst case is 0.0004 a.u. $/{ }^{\circ} \mathrm{C}$ for the range of higher temperatures. 


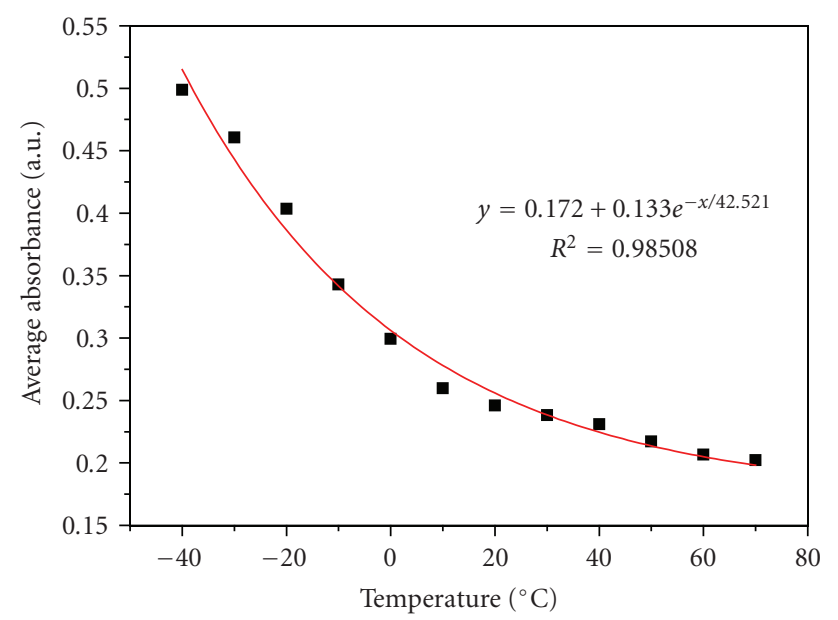

Figure 6: Average absorbance from 500 to $520 \mathrm{~nm}$ at different temperatures.

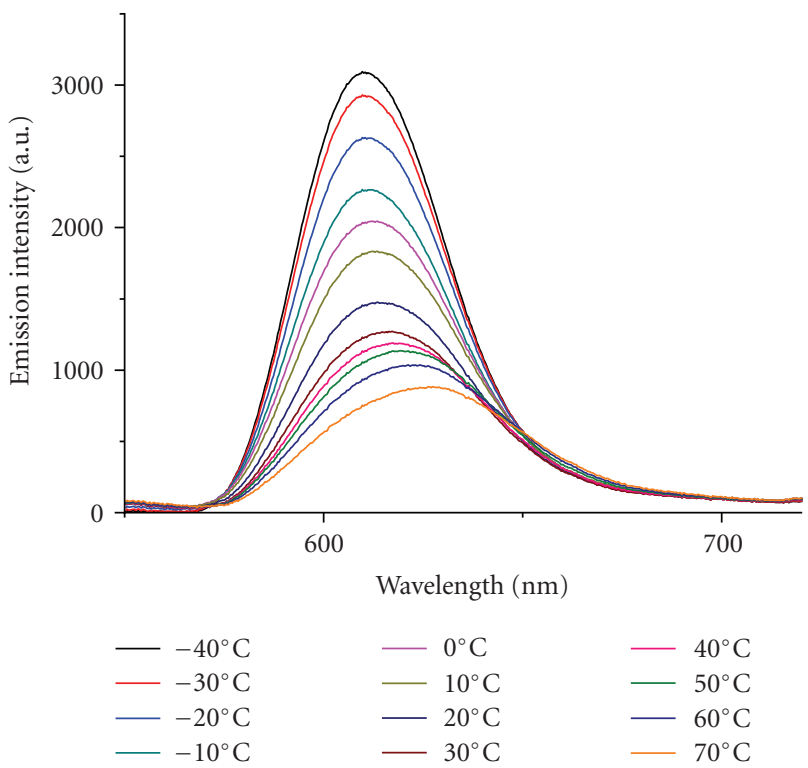

FIgURE 7: Fluorescent emission curves variation with temperature.

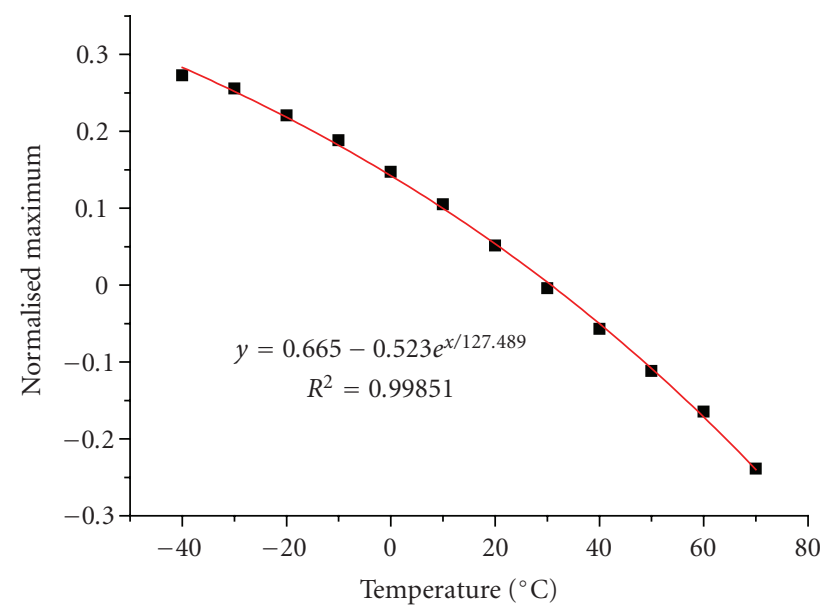

FIgURE 8: Normalized emission maxima.

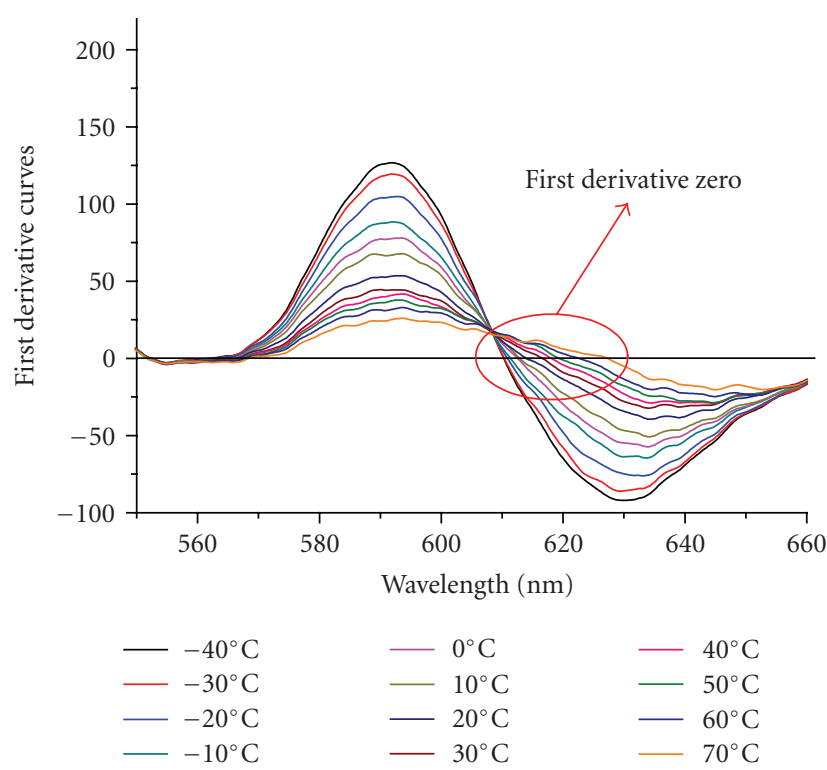

FIGURE 9: First derivative curves obtained from the emission spectra showed in Figure 5.

3.2. Emission Study. As it was previously commented, different emission properties of the sensor have been studied. With this aim, the QDs were excited using an Omnichrome Laser tuned at $470 \mathrm{~nm}$. The spectrometer registers the QD emission peak which, due to their quantum confinement properties, decreases in intensity when the temperature rises. This feature is shown in Figure 7, where each curve has been taken at different temperatures from -40 to $70^{\circ} \mathrm{C}$. It is important to realize that not only the maximum peak of the curve changes with the temperature. The wavelength and the width of this peak are increased as far as the temperature rises. Therefore, there are three features of the quantum dots emission peak which could be used to detect temperature variations: maximum values of each emission peak, wavelength of these values, and bandwidth of these peaks.

To study the maximum of the quantum dots emission peaks, a normalization method applied by Jorge et al. in a previous study [28] was utilized. It is based on taking two intensity signals $\left(S_{1}\right.$ and $\left.S_{2}\right)$ corresponding to two $5 \mathrm{~m}$ spectral windows on opposite sides of the emission peak and applying a normalization according to $\left(S_{1}-S_{2}\right) /\left(S_{1}+\right.$ $S_{2}$ ). The result of this normalization is proportional to the temperature and independent of the power fluctuations of the light source. In Figure 8, the evolution of this normalized maximum with temperature can be seen. These data can be approximated by an exponential signal with a correlation factor of $R^{2}=0.998$, rather better than the obtained absorption spectra measured in the precedent section. In some previous works $[26-28,30]$, the sensors apparently showed a linear behavior due to the narrower temperature range used. The average sensitivity of this sensor is $0.00465^{\circ} \mathrm{C}^{-1}$, and the sensitivity in the worst case is $0.001732^{\circ} \mathrm{C}^{-1}$ corresponding to the lower temperature values.

Another method to measure temperature with this sensor is based on monitoring the emission peak wavelength. The 


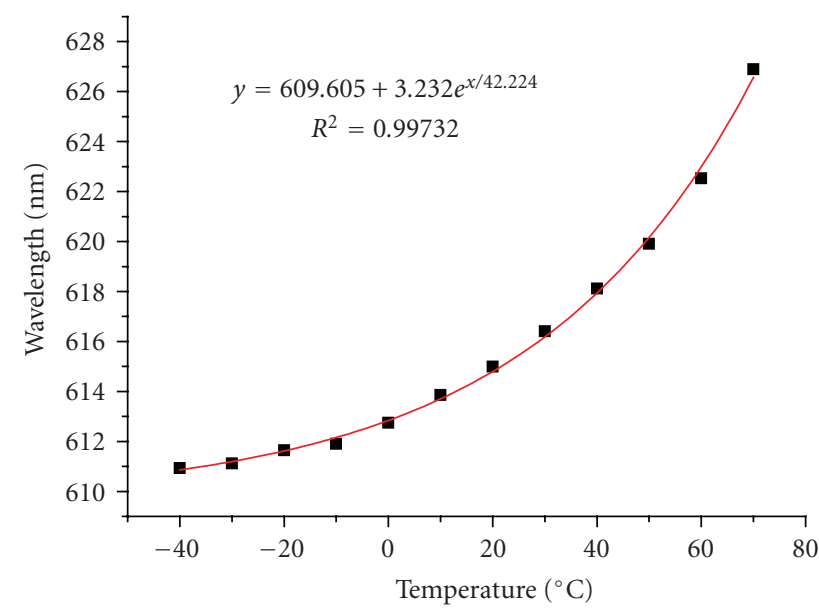

Figure 10: Emission peak wavelength at different temperatures.

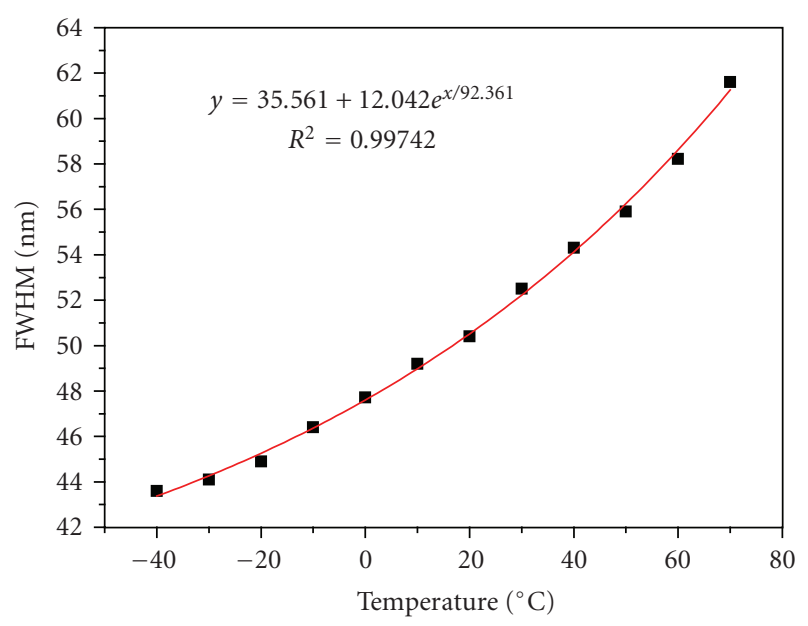

FIgURE 11: FWHM variation with temperature.

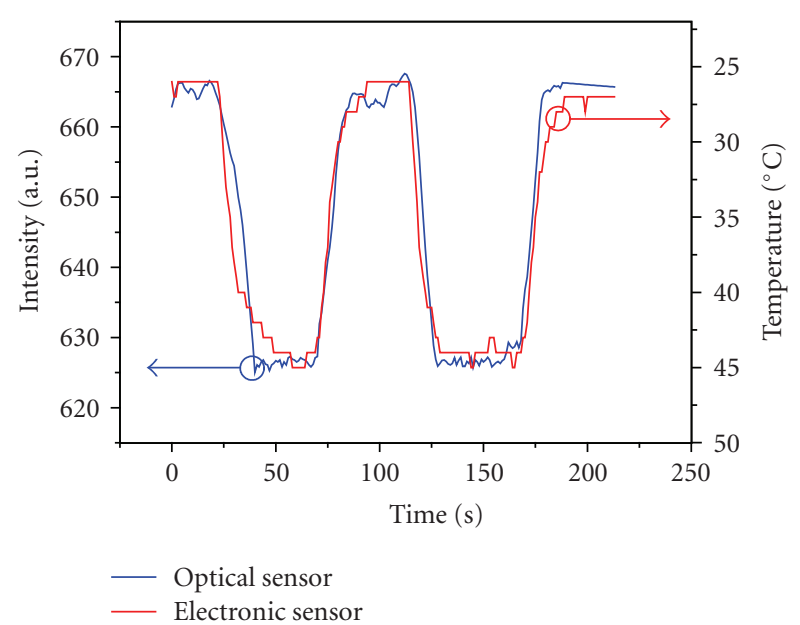

FIGURE 12: Dynamic behavior of the thermocouple (electronic) and the PCF QD-based (optical) sensors. The intensity measurement of $y$-axis is the average of the maximum intensity values between 600 and $640 \mathrm{~nm}$. emission peak shifts to higher wavelengths when the temperature rises, as is seen in Figure 7. Since the emission band does not provide a narrow peak to measure, it is necessary to develop a systematic method to calculate the emission peak wavelength, which matches with the maximum intensity. To reach this goal, a simple method based on the first derivative to detect the maxima of the emission curves is performed. Firstly, the derivative curves shown in Figure 9 are obtained from the graph in Figure 7. The first derivative curves reach the value of zero at the wavelengths which correspond to the maximum values of each emission spectrum as demonstrated in Figure 9. These wavelengths are plotted in Figure 10, having again an exponential fit with a good correlation factor of $R=0.99732$. The average sensitivity of this method is $0.1451 \mathrm{~nm} /{ }^{\circ} \mathrm{C}$, and the worst sensitivity is $0.0195 \mathrm{~nm} /{ }^{\circ} \mathrm{C}$ for the lower temperature range. The emission intensity and the emission peak wavelength give a lower sensitivity at lower temperatures whereas the absorption has the opposite behavior (higher sensitivity at lower temperatures). Combining the absorption with either the emission intensity or the peak wavelength, the sensitivity of these devices could be balanced in the whole range of temperatures.

In addition, a new emission feature was observed: the spectral width of the emission band shows temperature dependence. Taking advantage of this, the Full Width at Half Maximum (FWHM) of the emission band, a very popular parameter for the characterization of lasers, can be used as a sensing magnitude. The FWHM variation with temperature and its exponential approximation can be seen in Figure 10. The sensitivity of this method has an average value of $0.1636 \mathrm{~nm} /{ }^{\circ} \mathrm{C}$, and its minimum value of $0.05 \mathrm{~nm} /{ }^{\circ} \mathrm{C}$ corresponds to low temperatures. To our knowledge, this is the first time that the FWHM is used as a sensing parameter in QD-based sensors.

Finally, in order to verify for the dynamic behavior of the devices, the temperature sensor is subjected to sharp temperature changes between 25 and $45^{\circ} \mathrm{C}$. The QD sensor and a commercial sensor (a $\mathrm{K}$ thermocouple based device) are placed together and monitored at the same time to compare both responses. The QD emission magnitude measured is the average of the maximum intensity values between 600 and $640 \mathrm{~nm}$. In Figure 12 the responses of the QD sensor and the thermocouple are shown. The rise time and fall time of both devices are similar, as seen in the graph. They are also similar to the times observed in previous works by Bravo et al. [26].

\section{Conclusion}

In this work a temperature sensor has been fabricated by the deposition of quantum dot films inside the holes of a Photonic Crystal Fiber by means of the Layer-byLayer technique for the first time. The optical absorption, the emission intensity, and emission peak wavelength have shown a clear exponential behavior in the range from -40 to $+70^{\circ} \mathrm{C}$. It was observed that the measurement of the optical absorption can be used as a complementary method to improve the sensitivity at lower temperatures of the methods based on the emission properties. In addition, it has been 
proposed, for the first time in literature, the utilization of a parameter based on the spectral width of the emission band, the FWHM, in QD-based sensors.

\section{Acknowledgments}

This work was supported in part by the Spanish CICYTFEDER Research Grant TEC2006-12170/MIC. The authors are in debt to Jesus Arigita for all his previous experiments made for this project.

\section{References}

[1] T. A. Birks, P. J. Roberts, P. S. J. Russell, D. M. Atkin, and T. J. Shepherd, "Full 2-D photonic bandgaps in silica/air structures," Electronics Letters, vol. 31, no. 22, pp. 1941-1943, 1995.

[2] J. C. Knight, T. A. Birks, P. S. J. Russell, and D. M. Atkin, "All-silica single-mode optical fiber with photonic crystal cladding," Optics Letters, vol. 21, no. 19, pp. 1547-1549, 1996.

[3] H. L. Ho, Y. L. Hoo, W. Jin, et al., "Optimizing microstructured optical fibers for evanescent wave gas sensing," Sensors and Actuators B, vol. 122, no. 1, pp. 289-294, 2007.

[4] Y. L. Hoo, W. Jin, C. Shi, H. L. Ho, D. N. Wang, and S. C. Ruan, "Design and modeling of a photonic crystal fiber gas sensor," Applied Optics, vol. 42, no. 18, pp. 3509-3515, 2003.

[5] P. E. Hoiby, L. B. Nielsen, J. B. Jensen, T. P. Hansen, A. Bjarklev, and L. H. Pedersen, "Molecular immobilization and detection in a photonic crystal fiber," vol. 5317 of Proceedings of SPIE, pp. 220-223, 2004.

[6] W. Ye, L. L. Irene, C. R. Shuang, and P. Z. Jian, “Temperature sensor based on iodine-doped hollow core photonic crystal fiber," in Proceedings of the International Conference on Microwave and Millimeter Wave Technology (ICMMT'08), vol. 2, pp. 890-892, 2008.

[7] W. J. Bock, J. Chen, T. Eftimov, and W. Urbanczyk, "A photonic crystal fiber sensor for pressure measurements," IEEE Transactions on Instrumentation and Measurement, vol. 55, no. 4, pp. 1119-1123, 2006.

[8] V. P. Minkovich, D. Monzón-Hernández, J. Villatoro, and G. Badenes, "Microstructured optical fiber coated with thin films for gas and chemical sensing," Optics Express, vol. 14, no. 18, pp. 8413-8418, 2006.

[9] Y. Ruan, T. C. Foo, S. Warren-Smith, et al., "Antibody immobilization within glass microstructured fibers: a route to sensitive and selective biosensors," Optics Express, vol. 16, no. 22, pp. 18514-18523, 2008.

[10] G. Decher, "Fuzzy nanoassemblies: toward layered polymeric multicomposites," Science, vol. 277, no. 5330, pp. 1232-1237, 1997.

[11] M. T. Crisp and N. A. Kotov, "Preparation of nanoparticle coatings on surfaces of complex geometry," Nano Letters, vol. 3, no. 2, pp. 173-177, 2003.

[12] F. J. Arregui, I. R. Matías, and R. O. Claus, "Optical fiber sensors based on nanostructured coatings fabricated by means of the layer-by-layer electrostatic self-assembly method," vol. 6619 of Proceedings of SPIE, 2007.

[13] J. Goicoechea, F. J. Arregui, J. M. Corres, and I. R. Matias, "Study and optimization of self-assembled polymeric multilayer structures with neutral red for $\mathrm{pH}$ sensing applications," Journal of Sensors, vol. 2008, Article ID 142854, 7 pages, 2008.

[14] R. K. Iler, "Multilayers of colloidal particles," Journal of Colloid and Interface Science, vol. 21, no. 6, pp. 569-594, 1966.
[15] A. P. Alivisatos, "Semiconductor clusters, nanocrystals, and quantum dots," Science, vol. 271, no. 5251, pp. 933-937, 1996.

[16] J. M. Costa-Fernandez, "Optical sensors based on luminescent quantum dots," Analytical and Bioanalytical Chemistry, vol. 384, no. 1, pp. 37-40, 2006.

[17] D. C. Oertel, M. G. Bawendi, A. C. Arango, and V. Bulović, "Photodetectors based on treated CdSe quantum-dot films," Applied Physics Letters, vol. 87, no. 21, pp. 1-3, 2005.

[18] T. J. Bukowski and J. H. Simmons, "Quantum dot research: current state and future prospects," Critical Reviews in Solid State and Materials Sciences, vol. 27, no. 3-4, pp. 119-142, 2002.

[19] I. L. Medintz, H. T. Uyeda, E. R. Goldman, and H. Mattoussi, "Quantum dot bioconjugates for imaging, labelling and sensing," Nature Materials, vol. 4, no. 6, pp. 435-446, 2005.

[20] A. R. Clapp, E. R. Goldman, H. Tetsuo Uyeda, E. L. Chang, J. L. Whitley, and I. L. Medintz, "Monitoring of enzymatic proteolysis using self-assembled quantum dot-protein substrate sensors," Journal of Sensors, vol. 2008, Article ID 797436, 10 pages, 2008.

[21] M. Bruchez Jr., M. Moronne, P. Gin, S. Weiss, and A. P. Alivisatos, "Semiconductor nanocrystals as fluorescent biological labels," Science, vol. 281, no. 5385, pp. 2013-2016, 1998.

[22] V. L. Colvin, M. C. Schlamp, and A. P. Alivisatos, "Lightemitting diodes made from cadmium selenide nanocrystals and a semiconducting polymer," Nature, vol. 370, no. 6488, pp. 354-357, 1994.

[23] P. Jorge, M. A. Martins, T. Trindade, J. L. Santos, and F. Farahi, "Optical fiber sensing using quantum dots," Sensors, vol. 7, no. 12, pp. 3489-3534, 2007.

[24] K. E. Sapsford, T. Pons, I. L. Medintz, and H. Mattoussi, "Biosensing with luminescent semiconductor quantum dots," Sensors, vol. 6, no. 8, pp. 925-953, 2006.

[25] G. W. Walker, V. C. Sundar, C. M. Rudzinski, A. W. Wun, M. G. Bawendi, and D. G. Nocera, "Quantum-dot optical temperature probes," Applied Physics Letters, vol. 83, no. 17, pp. 3555-3557, 2003.

[26] J. Bravo, J. Goicoechea, J. M. Corres, F. J. Arregui, and I. R. Matias, "Encapsulated quantum dot nanofilms inside hollow core optical fibers for temperature measurement," IEEE Sensors Journal, vol. 8, no. 7, pp. 1368-1374, 2008.

[27] G. De Bastida, F. J. Arregui, J. Goicoechea, and I. R. Matias, "Quantum dots-based optical fiber temperature sensors fabricated by layer-by-layer," IEEE Sensors Journal, vol. 6, no. 6, pp. 1378-1379, 2006.

[28] P. A. S. Jorge, M. Mayeh, R. Benrashid, P. Caldas, J. L. Santos, and F. Farahi, "Quantum dots as self-referenced optical fibre temperature probes for luminescent chemical sensors," Measurement Science and Technology, vol. 17, no. 5, pp. 10321038, 2006.

[29] F. J. Arregui, I. R. Matias, J. Goicoechea, and I. del Villar, Sensors Based on Nanostructured Materials, Springer, New York, NY, USA.

[30] M. L. Redígolo, W. A. Arellano, L. C. Barbosa, C. H. Brito Cruz, C. L. Cesar, and A. M. De Paula, "Temperature dependence of the absorption spectra in CdTe-doped glasses," Semiconductor Science and Technology, vol. 14, no. 1, pp. 5863, 1999.

[31] C. Sifuentes, Y. O. Barmenkov, A. N. Starodumov, V. N. Filippov, and A. A. Lipovskii, "Application of CdSe-nanocrystallitedoped glass for temperature measurements in fiber sensors," Optical Engineering, vol. 39, no. 8, pp. 2182-2186, 2000. 

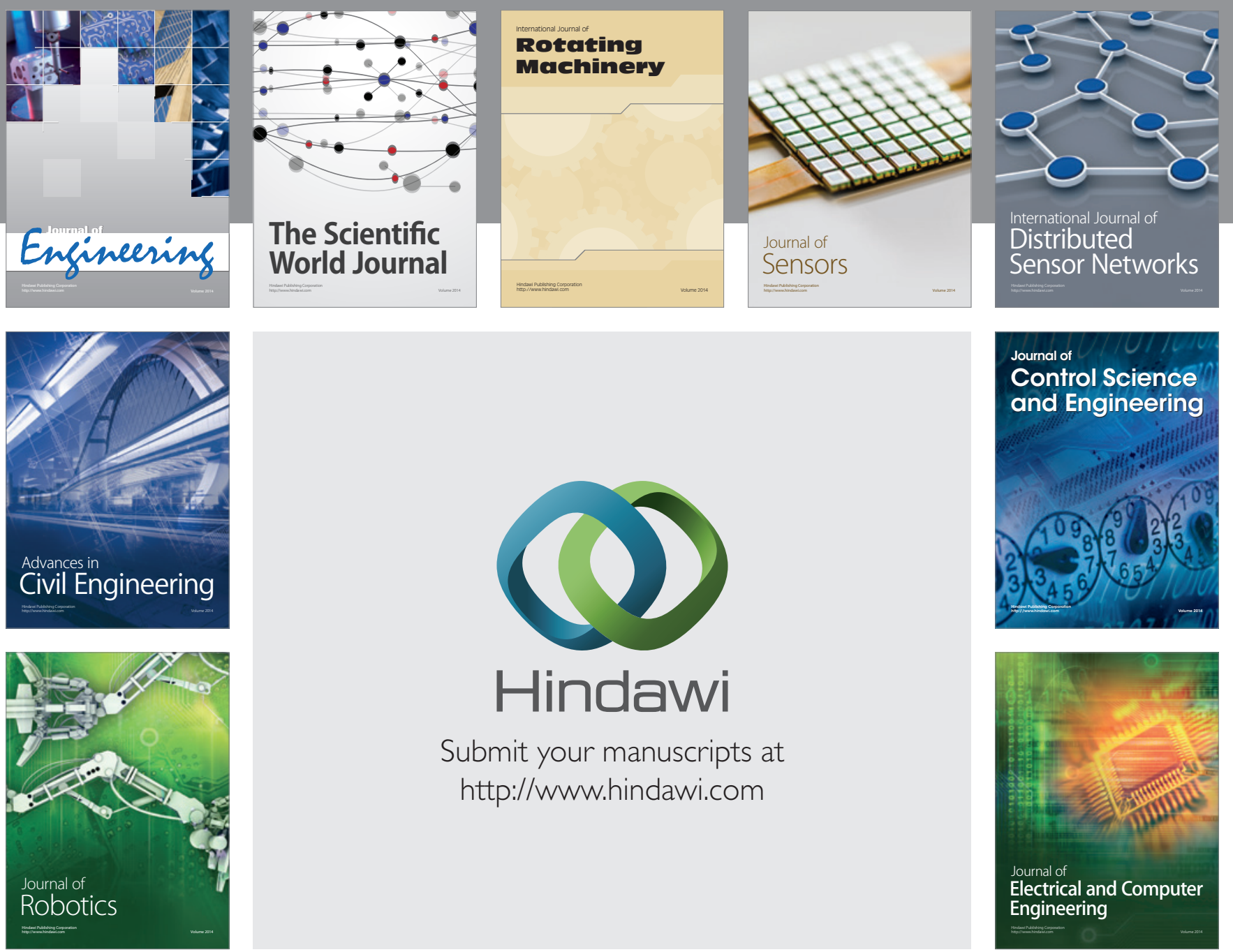

Submit your manuscripts at

http://www.hindawi.com
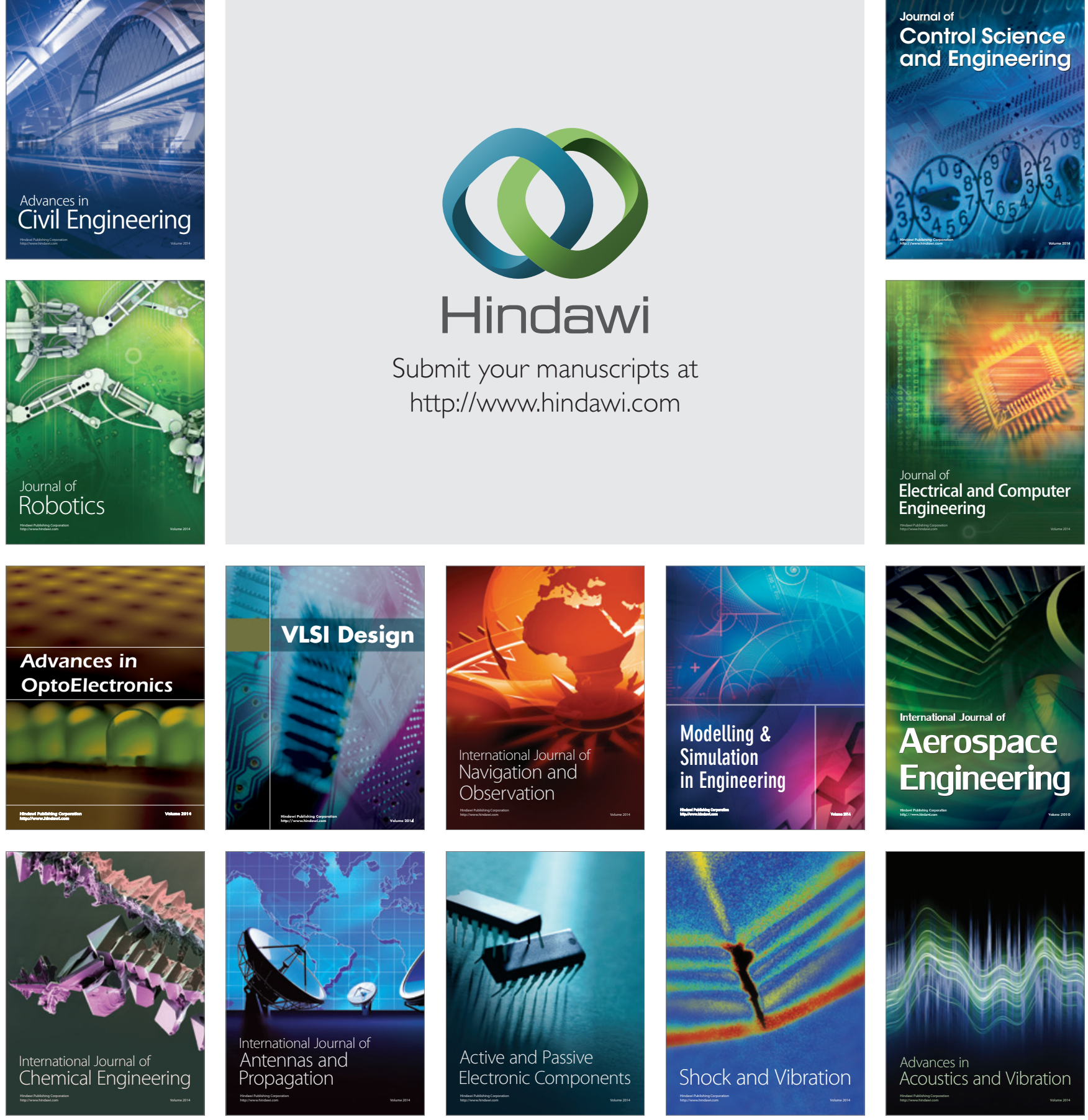